\title{
Transoral rotational esophagogastric fundoplication: technical, anatomical, and safety considerations
}

\author{
Reginald C. W. Bell • Guy-Bernard Cadière
}

Received: 14 June 2010/ Accepted: 27 November 2010/Published online: 24 December 2010

(C) The Author(s) 2010. This article is published with open access at Springerlink.com

\begin{abstract}
Background Gastroesophageal reflux disease (GERD) results primarily from the loss of an effective antireflux barrier, which forms a mechanical barrier against the retrograde movement of gastric content. Restoration of the incompetent antireflux barrier is possible by longitudinal and rotational advancement of the gastric fundus about the lower esophagus, creating an esophagogastric fundoplication. This article describes the technique of performing a rotational and longitudinal esophagogastric fundoplication, performed transorally using EsophyX.

Methods The transoral incisionless fundoplication (TIF) technique enables the creation of a full-thickness esophagogastric fundoplication with fixation extending longitudinally up to $3.5 \mathrm{~cm}$ above the Z-line and rotationally more than 270 degrees around the esophagus. A key element of the technique involves rotating the fundus around the esophagus with a tissue mold during gastric desufflation. Anatomic considerations and use of the device's tissue invaginator to push the esophagus caudally are important to ensure safe positioning of the plications below the diaphragm. The steps of the technique are described in detail, and suggestions are given about patient selection and care, as well as prevention and management of complications.
\end{abstract}

R. C. W. Bell ( $\square)$

Swedish Medical Center \& SurgOne, P.C, 400 W Hampden

Place, Suite 230, Englewood, CO 80110, USA

e-mail: rbell@surgone.com

G.-B. Cadière

Department of Gastrointestinal Surgery, Centre Hospitalier

Universitaire St. Pierre, Brussels, Belgium
Keywords Antireflux surgery - Endoscopic - EsophyX . Gastroesophageal reflux disease · Transoral incisionless fundoplication

A novel endoluminal plicating device (EsophyX) was developed to reconstruct the antireflux barrier and treat gastroesophageal reflux disease (GERD). The technique has evolved through clinical trials conducted since 2006 and more than 4,000 cases performed worldwide [1-3]. The purpose of this paper was to codify the technique whereby a full thickness esophagogastric fundoplication is created using this device, to describe potential complications, and to delineate the relationship between these complications and human anatomy to minimize the potential for complications. The basis for this description is experience with more than 100 procedures beginning in November 2008. The clinical outcomes of these patients are reported elsewhere [4]. Because the procedure is increasing in popularity, we think that it is important to describe the technique with an emphasis on anatomy and safety. The steps of the technique are described in detail below and are summarized in Table 1. Suggestions are given about patient selection and care, as well as prevention and management of complications.

\section{Methods}

The EsophyX device (EndoGastric Solutions, Inc., Redmond, WA, USA; Fig. 1) is introduced into the stomach transorally over a flexible endoscope. The device enables molding of tissue and placement of polypropylene suture material in the region of the gastroesophageal (GE) junction. It is composed of a handle, wherein the various 
Table 1 Steps to the TIF procedure

\begin{tabular}{|c|c|}
\hline Preoperative endoscopy & $\begin{array}{l}\text { Height hiatal hernia } \leq 2 \mathrm{~cm} \text {, reduces fully } \\
\text { Transverse dimensions of hiatus }<3 \mathrm{~cm} \text { max }\end{array}$ \\
\hline Preoperative medications & Antiemetics, antibiotics, and anticholinergics \\
\hline Anesthesia & General endotracheal \\
\hline Position & Semirecumbent \\
\hline Initial endoscopy & $\begin{array}{l}\text { Transverse dimensions of hiatus }<3 \mathrm{~cm} \text { max } \\
\text { Record distance from incisors to hiatal landmark }\end{array}$ \\
\hline Device introduction & Tissue mold handle to left shoulder orients elbow of tissue mold to course of pharynx \\
\hline Device retroflexion & Direct vision with endoscope retroflexed. \\
\hline $\mathrm{CO}_{2}$ insufflation & Through working channel, pressure $12-15 \mathrm{mmHg}$ \\
\hline Identify anatomic landmarks & Lesser curve (12 o'clock); greater curve (6 o'clock) \\
\hline Initial helical screw deployment & 12 o'clock insertion at Z-line/GE junction \\
\hline Three anterior rotational plication sets & $\begin{array}{l}\text { Roll tissue from } 6 \text { o'clock anteriorly toward } 1 \text { o'clock with tissue mold; tension on helical } \\
\text { retractor; gastric desufflation } \\
\text { Lock helical retractor and tissue mold; apply suction to tissue invaginator for } 30 \mathrm{~s} \\
\text { Advance device to within } 1 \mathrm{~cm} \text { of distance corresponding to measured distance to hiatal } \\
\text { landmark; rotate device out of corner to align tissue mold } \\
\text { Advance stylet furthest from corner (posterior in this case) first, deploy fastener } \\
\text { Complete first rotational plication set by advancing another stylet and deploy second fastener } \\
\text { Create two additional anterior rotational plication sets at slightly different depths. This will } \\
\text { create plications from } 2 \text { o'clock to } 4 \text { o'clock at depths up to } 3 \mathrm{~cm}\end{array}$ \\
\hline $\begin{array}{l}\text { Rotate tissue mold through lesser curve to } \\
\text { posterior corner }\end{array}$ & $\begin{array}{l}\text { Advance device with helical retractor cable slack, tissue mold partially closed, and rotate device } \\
\text { counterclockwise }\end{array}$ \\
\hline Three posterior rotational plication sets & $\begin{array}{l}\text { Similar to the anterior plication sets, but rotation is now clockwise from } 6 \text { o'clock toward } 11 \\
\text { o'clock, and the anterior stylet is advanced first. Three plication sets will be created from } 7 \\
\text { o'clock to } 10 \text { o'clock at different depths up to } 3 \mathrm{~cm}\end{array}$ \\
\hline
\end{tabular}

Rotate tissue mold back through lesser curve back to anterior corner

Two anterior longitudinal plication sets at $12: 30$ to 2 o'clock

Gentle longitudinal advanced caudally with the helical retractor and infolding of tissue with the tissue mold during gastric desufflation to create two anterior longitudinal plication sets of $1-2 \mathrm{~cm}$ depth

Reposition helical retractor to 4 o'clock

This is the second helical retractor placement and is done to aid in caudal retraction for the final longitudinal plication

One greater curve longitudinal plication set at This plication set must be performed carefully with attention to the location of the diaphragm 5 o'clock

One additional plication set

Remove device

Final endoscopy

Postoperative care

\section{As needed}

Release helical retractor and pull back into tissue mold

Straighten tissue mold under direct visualization

Remove device while observing esophagus with endoscope just inside device. Helical retractor should be pulled back; tissue mold knob externally to left shoulder

Assess plication; assess for bleeding or perforation

Pain medication as needed. GI cocktail: viscous Lidocaine, Donnatal, antacids

Continue PPIs for 2 weeks. Clear to full liquid diet without carbonation. Consider water-soluble contrast study before discharge controls are located, a chassis of $18 \mathrm{~mm}$ in diameter through which the endoscope is inserted and control channels run, side holes on the distal end of the shaft to which external suction can be applied (the tissue invaginator), a tissue mold, which when brought into retroflexion pushes tissue against the shaft of the device, a helical screw, which is advanced into tissue to pull tissue caudally between the tissue mold and the shaft, two stylets, which advance from the shaft of the device through the plicated tissue and then through eyelets in the tissue mold, and a cartridge containing polypropylene $\mathrm{H}$-shaped fasteners (or plicators), which are deployed over the stylets so that the trailing leg engages within the esophageal lumen and the leading leg engages within the gastric lumen. 
Fig. 1 EsophyX device. General view of the device (top left) and close views of the working end

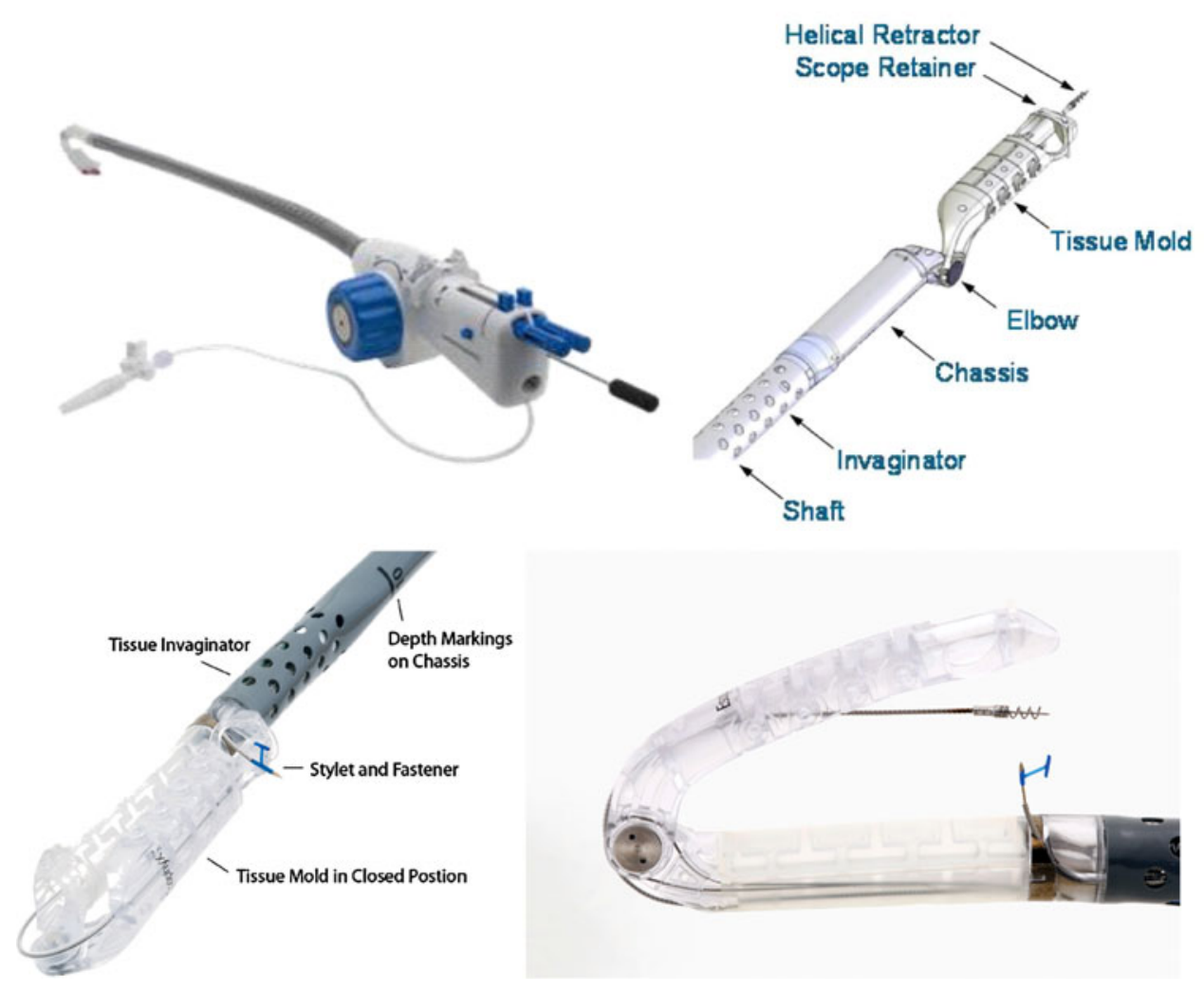

Technique evolution

Initial descriptions of the transoral incisionless fundoplication (TIF) technique involved reduction of any hiatal hernia by gripping the esophagus with the chassis' tissue invaginator and advancing the device caudally, and then creation of a full-thickness gastrogastric plication at the level of the Z-line (Fig. 2A). The helical retractor was used to pull full-thickness gastric wall caudally into the tissue mold. The mold apposed the two layers of gastric wall and sandwiched between them the phrenoesophageal membrane, which could be demonstrated in the canine model and observed in human cases [5]. Subsequently, polypropylene fasteners were deployed over their respective stylets. This created a partially circumferential fundoplication but was limited to gastric tissue, and was dubbed "ELF"endoluminal fundoplication — and later "TIF."

Using a canine model, Jobe et al. described the technique of creating a transoral esophagogastric fundoplication with rotational and longitudinal elements using the EsophyX device (Fig. 2B). This new technique was dubbed "TIF 2," with the gastrogastric technique now referred to as "TIF 1" [5]. The TIF 2 procedure was demonstrated by manometric vector volume analysis to be superior to the TIF 1 technique and similar (in the canine model) to the Nissen fundoplication [5].

The use of certain device components has evolved with these techniques. The earliest use of the helical retractor was simply to retract tissue caudally. However, the multifunctional helix also may be used as an anchor toward which the tissue mold pushes tissue. The tissue mold can be used as a stop, ensuring that the $\mathrm{H}$-fasteners deploy properly. It can be used to bring tissue into apposition to create an esophagogastric plication. In conjunction with the anchoring effect of the helical screw, the tissue mold can be used to rotate gastric fundic tissue radially around the distal esophagus.

This report of the TIF 2 procedure in humans owes much to the technique developed by Blair Jobe and Stefan Kraemer [5]. We have altered the technique by deploying the helical retractor at the lesser curve position only once to maximize the rotational effect. The use of the tissue invaginator to fix the device chassis to the esophagus also has been modified. Applying suction before closure of the tissue mold (TIF 1) enabled reduction of any hiatal hernia, whereas our technique of applying suction after closure of the tissue mold allows for both caudal advancement of the esophagus, enabling an intra-abdominal esophagogastric plication, and rotation of the esophagus, providing better visualization of stylet advancement.

\section{The TIF technique}

\section{Preoperative care}

Intravenous antibiotics are administered preoperatively as the plications are deployed transmurally. Proton pump 
Fig. 2 A TIF 1 procedure with gastrogastric plications placed at the level of the Z-line. B TIF 2 technique creates an esophagogastric fundoplication proximal to the $\mathrm{Z}$-line a
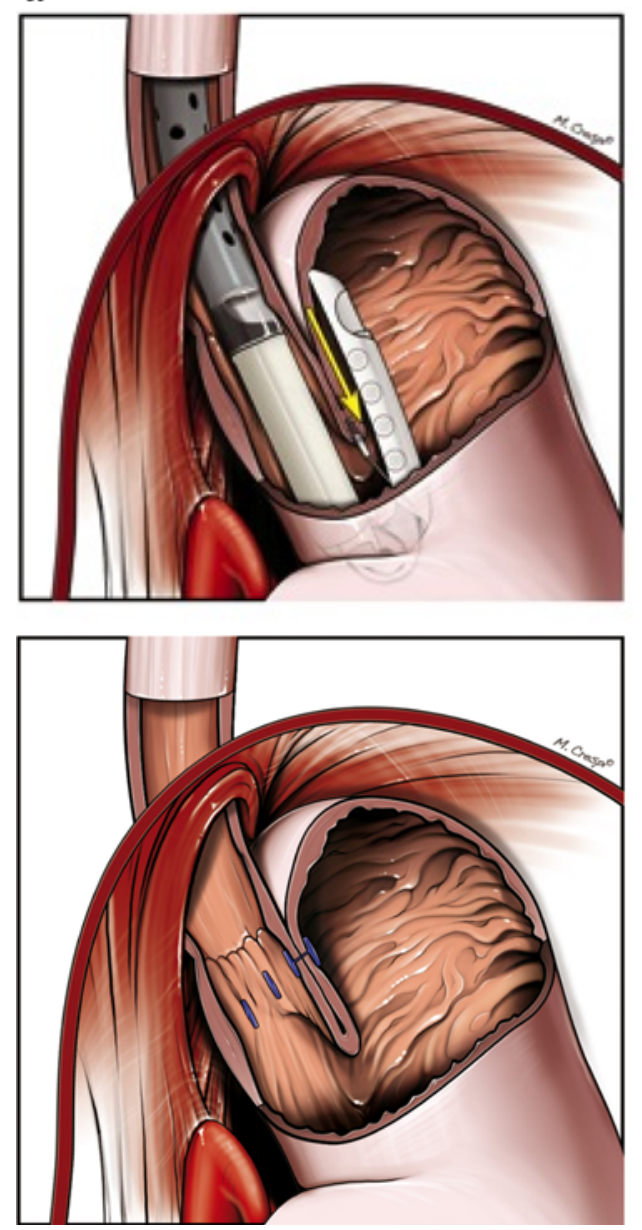

b
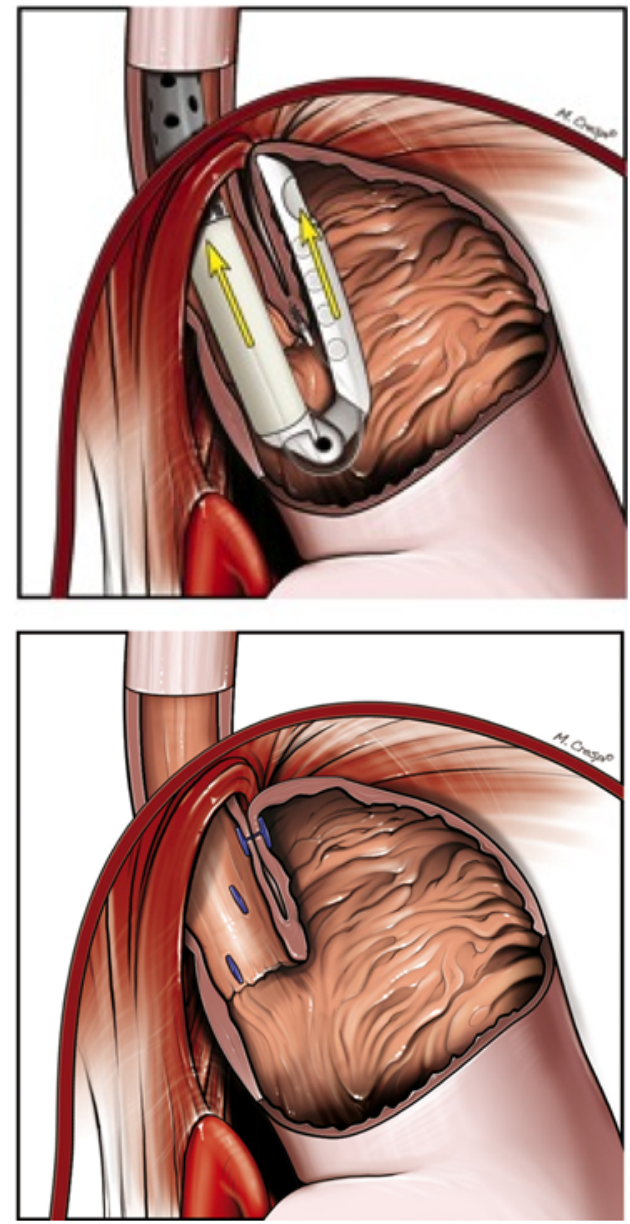

inhibitor (PPI) therapy is administered preoperatively and then continued for 14 days postoperatively to avoid hemorrhage from the gastric side of the fundoplication. Medications to reduce nausea are started preoperatively and continued postoperatively for $24 \mathrm{~h}$ or as needed. Anticholinergics to reduce mucus secretion also are given.

\section{Surgical care}

It is feasible to perform the procedure on a gurney rather than an operating room table. The patient is placed mostly supine to lessen pressure on the GE junction from the liver (Fig. 3). The procedure is performed under general endotracheal anesthesia with an emphasis on complete diaphragm relaxation. This often is difficult to communicate to the anesthetist who is looking for twitches on a skinapplied monitor; our experience indicates that the diaphragm requires more muscle relaxant than other muscles. During the procedure, difficult insufflation of the stomach or the presence of hiccups is indicative of incomplete relaxation. Oral tracheal intubation avoids potential epistaxis, which can obscure the operative field; however, nasotracheal intubation may be useful in patients with a small hypopharynx, such as pediatric patients.

Preoperative endoscopy under general anesthesia is necessary to determine: (1) the distance (in centimeters) between the incisor teeth and the diaphragmatic hiatus, and (2) the greatest transverse dimension (in centimeters) of the hiatus at the GE junction under full gastric distention and complete diaphragmatic relaxation.

Understanding the relationship between the esophagus, esophageal hiatus, and diaphragm is critical to the safe advancement and deployment of fasteners below the diaphragm. Because the esophagogastric fundoplication places fasteners above the Z-line, there is a potential for esophageal laceration, esophageal leak, mediastinal infection, or subdiaphragmatic infection. The diaphragm can be incorporated into the plication, and subsequent tension on the fasteners with diaphragm movement may pull the fasteners through the esophageal wall, leading to mediastinitis, or the fundus wall, leading to subdiaphragmatic infection. The end of the tubular esophagus with the hernia fully reduced and the stomach partially insufflated is in our experience the most reproducible landmark for the caudal 
Fig. 3 The liver compresses the gastroesophageal junction less with the patient in supine than in the left lateral decubitus position

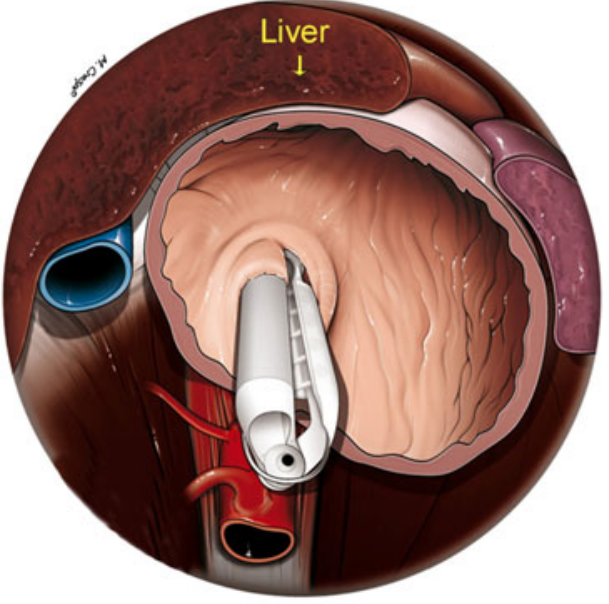

Patient Supine

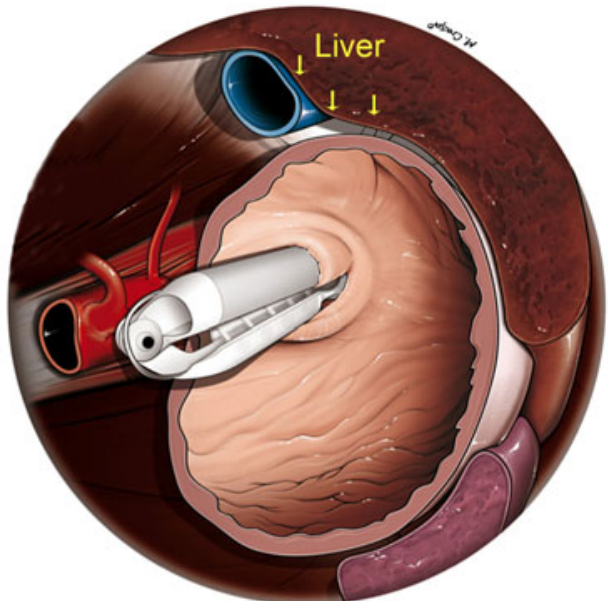

Patient Left Decubitus edge of the diaphragmatic hiatus and we refer to this as the "hiatal landmark." The measured distance from the incisor teeth to the hiatal landmark is one method of locating the diaphragm during the TIF procedure. A piece of brightly colored tape is placed on the shaft of the device at the number corresponding to this measurement as a ready reminder of this distance. If the number on the outside of the EsophyX device at the teeth is equal to or greater than the hiatal landmark location (in centimeters), it is likely that the esophagogastric plication will be within the abdomen and that the fasteners will not traverse the mediastinal or pleural space or incorporate the diaphragm.

Additionally, we note the largest transverse dimensions of the hiatal opening both at preoperative endoscopy and at intraoperative pre-TIF endoscopy. A retroflexed view with generous gastric distention frequently demonstrates the edges of the crura impinging on the gastroesophageal junction (Fig. 4). We have found that patients in whom the transverse dimensions of the hiatus are $<3 \mathrm{~cm}$ (roughly 3 times the diameter of the endoscope) can easily have the TIF procedure performed. When transverse hiatal dimensions are $>3 \mathrm{~cm}$, there is a chance that the fundoplication will end up in the thorax, which may not be desirable.

\section{Device introduction}

Hypopharyngeal perforations, described early in the TIF experience [1], underline the caution needed in introducing the device. Esophageal dilatation with a 56-60-Fr Maloney or Savory dilator before device introduction may aid in smooth placement and ensure that the device can be introduced before unpackaging the device. Occasionally rolling the patient to the left decubitus position or placing the head in the sword-swallowing position may help.

Once the device is introduced, the stomach is insufflated. The use of $\mathrm{CO}_{2}$ from a laparoscopic insufflator at

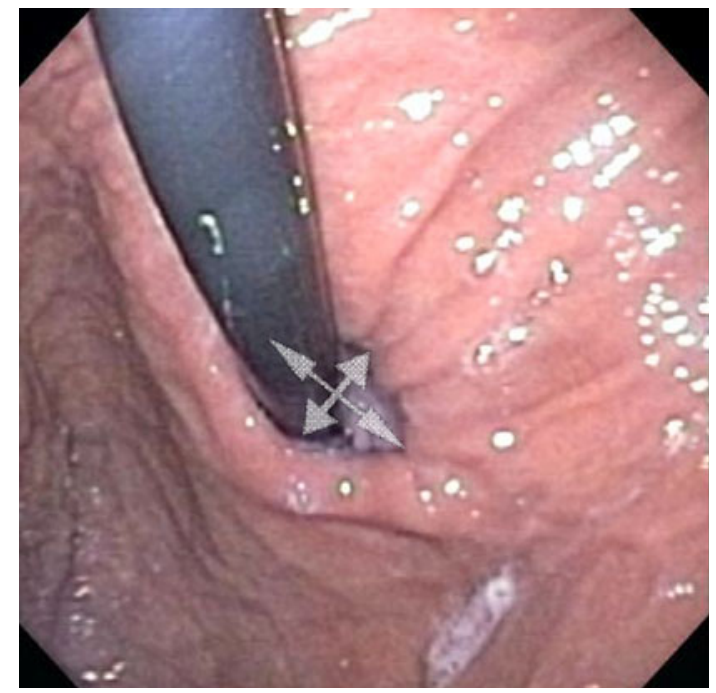

Fig. 4 Retroflex view of gastroesophageal junction. Transverse dimensions of hiatus are marked with crossing arrows

15-18 $\mathrm{mmHg}$ through the working channel of the flexible endoscope increases the flow and leads to a faster and more sustained gastric insufflation. The gastroscope is placed in retroflexion, and the tissue mold is retroflexed under direct visualization.

\section{Definition of terms and anatomic relationships}

Anatomic relations both within the stomach and to external organs, such as liver, spleen, aorta, heart, and left and right crura, are not always self-evident during flexible upper gastrointestinal endoscopy. To aid with orientation, we propose a terminology using the clock face. On endoscopy in retroflex view the 12 o'clock position is defined as the location of the lesser curve. Six o'clock refers to the valve position, which faces the greater curve of the stomach (Fig. 5). 


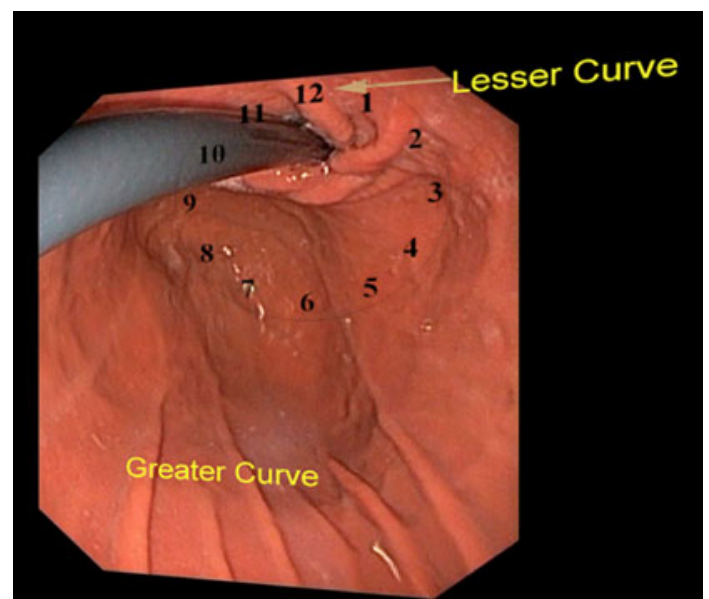

Fig. 5 Retroflex endoscopic view of gastroesophageal valve with clock face annotations. The lesser curve is defined as 12 o'clock

The relationship of the stomach to aorta, vena cava, spleen, heart, liver, and diaphragm is important to understand (Fig. 6). The tissue mold can cause trauma to the spleen during retroflexion and opening. Incorrect advancement of the stylet out of alignment with the tissue mold could potentially injure the liver, diaphragm, heart, or great vessels. The diaphragm ventral to the esophageal hiatus runs in a relatively transverse direction (Fig. 7). Along the left edge of the esophageal hiatus, however, the diaphragm courses superolaterally paralleling the fundus (Fig. 8). Failure to recognize this transition can lead to closure of the tissue mold so that it incorporates the diaphragm lateral to the crus, with subsequent stylet and fastener deployment through the diaphragm.

\section{Definitions}

Rotation directions Directions are described relative to the video screen with the endoscope in retroflex view. To obtain a counterclockwise rotation of the device on the screen, the device is rotated externally clockwise along its axis of insertion.
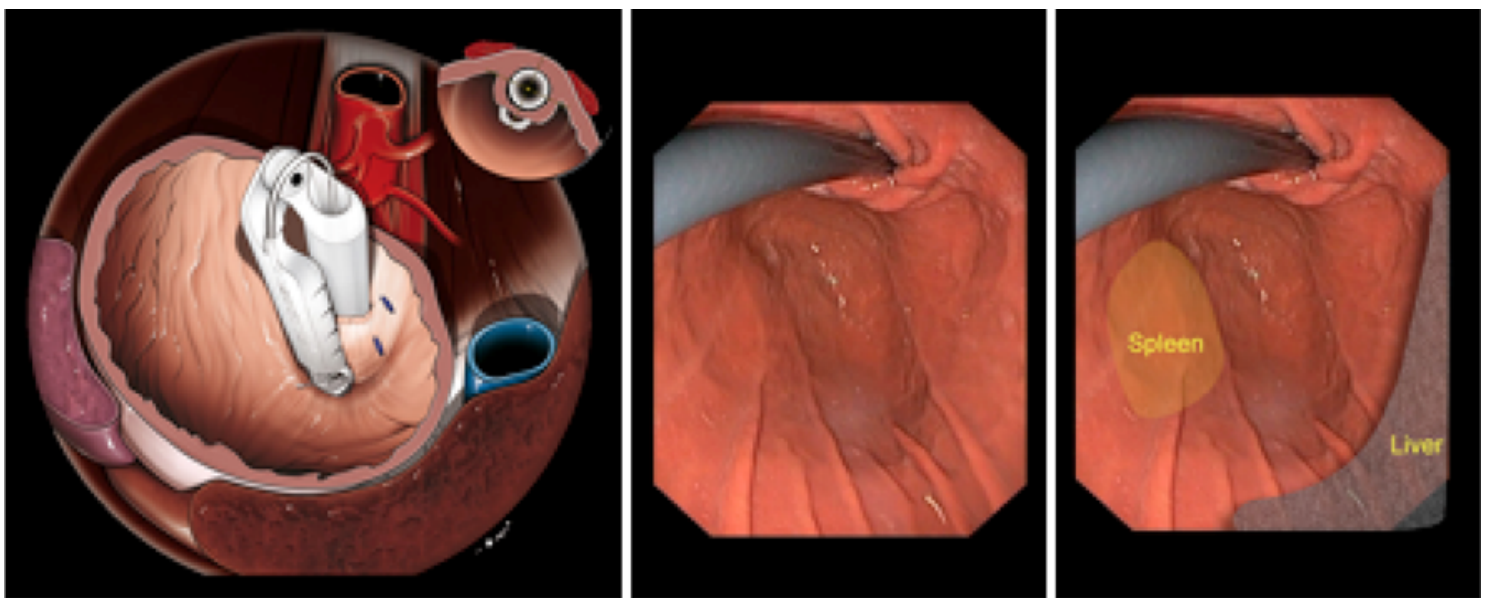

Fig. 6 External relations of aorta, vena cava, liver, and spleen from a standard endoscopic view

Fig. 7 The course of the diaphragm (dotted lines) both in anatomic transverse plane (left) and typical endoscopic view (right)
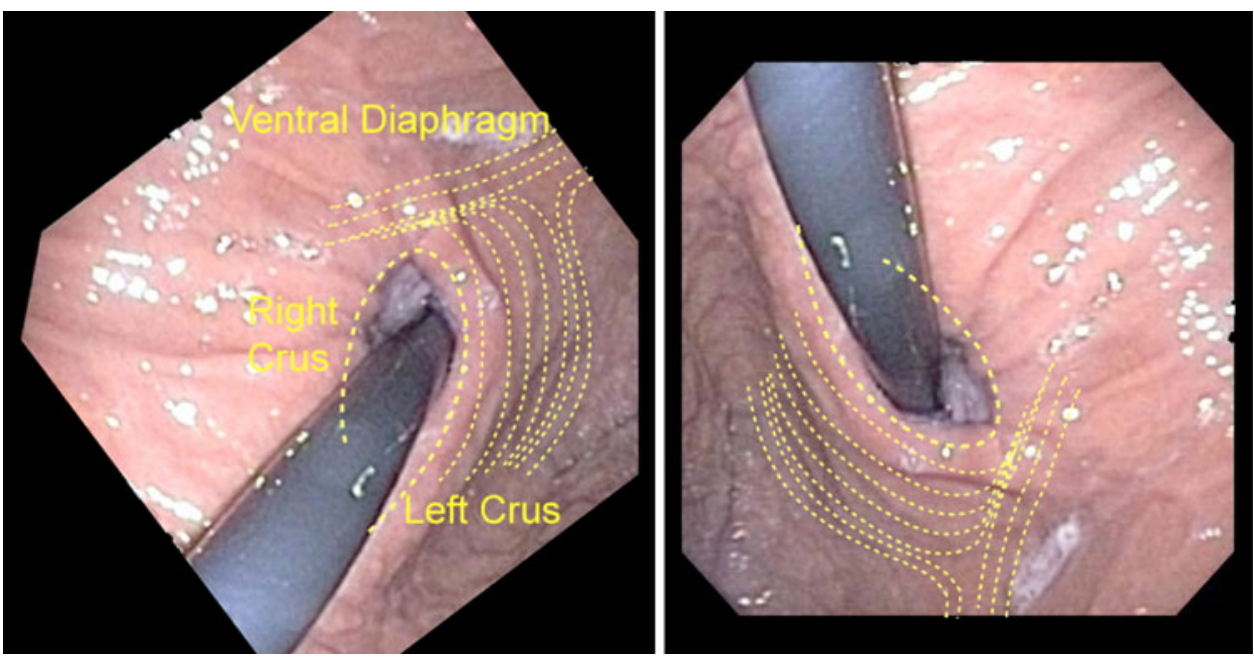
Fig. 8 Endoscopic view (left) indicating anterior corner and the location of the diaphragm (lines) where it follows the superolateral course of the fundus. Schematic drawing of laparoscopic view (right) depicting the position of diaphragmatic hiatus in relationship to the fundus, crura, and the gastroesophageal junction. Caution is needed not to incorporate the diaphragm in plication (arrow)

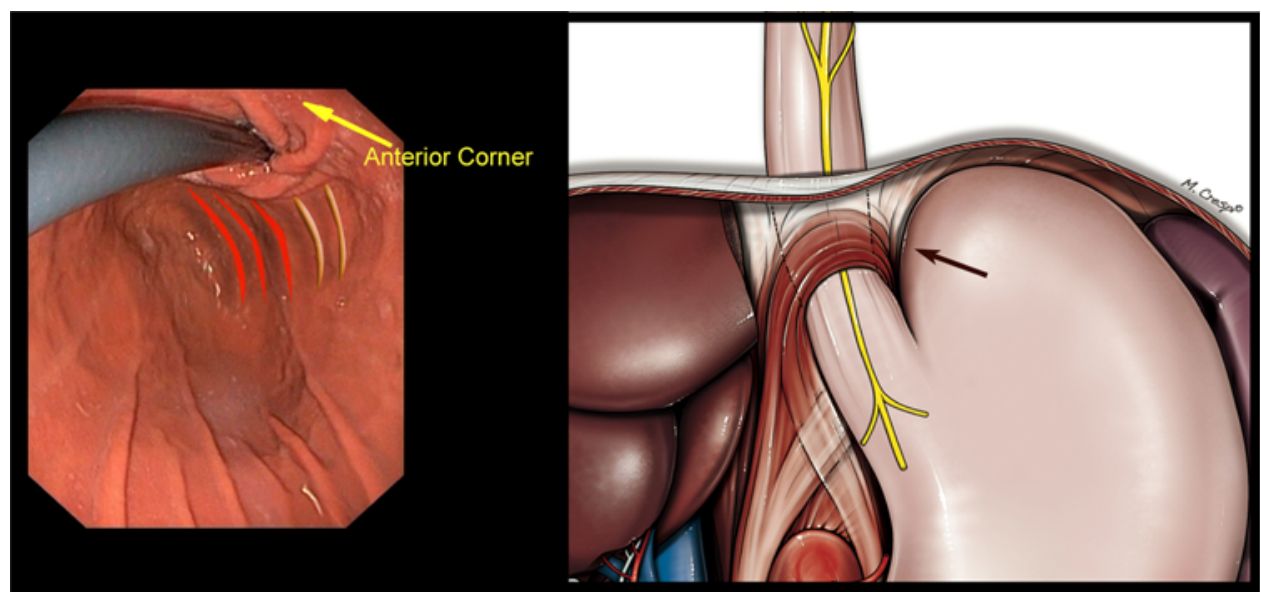

Plication set When one fastener properly deploys over the anterior stylet and one fastener properly deploys over the posterior stylet.

Position The midpoint of the lesser curve is defined as 12 o'clock and the rim of the GE valve is considered a clock face. Positions are described relative to the 12 o'clock position, e.g., the greater curve would be at 6 o'clock.

Plicator position Because a plication set deploys two fasteners at the same depth but at different radial distances from the 12 o'clock position, the plicator position is considered for the fastener that is closest to the lesser curve (12 o'clock position).

Plicator depth The distance the plicator set is cranial to the Z-line observed during the procedure with the endoscope in retroflexion. (We do not use the clear port in the device to ascertain the Z-line with the scope antegrade and withdrawn as was done in TIF 1.)

\section{Plication}

I. Helix placement at 12 o'clock and initial anterior rotational plication sets:

a. Once the tissue mold on the EsophyX device is retroflexed, the tissue mold is closed against the device and the device is rotated counterclockwise to the 12 o'clock position. The device is then pulled back so that the tip of the tissue mold goes just inside the esophageal lumen. At this point, the helical screw is advanced into the esophagogastric junction right at the Z-line. Slight opening of the tissue mold enables the operator to visualize the helical screw engaging at the Z-line. If the Z-line is irregular, the helical screw is engaged just at the start of the gastric folds at the EG junction. The shaft of the device is then advanced caudally a few centimeters and rotated clockwise on the screen, so that the tissue mold can be opened and the helical screw cable freed from the tissue mold. The tissue mold is rotated to the 6 o'clock position (greater curve) and partially closed.

b. The following three steps are executed simultaneously to accomplish the so called "Bell Roll maneuver" (Fig. 9) to execute a rotational plication set:

1. The tissue mold is brought to a partially closed position against the fundus of the stomach and the device is pulled back (cranially) $1-3 \mathrm{~cm}$, depending on the depth of plication intended.

2. Tension is applied to the helical retractor to advance the GE junction caudally.

3. The stomach is desufflated by the endoscopist.

4. While the stomach is being desufflated, the tissue mold is opened slightly (a few millimeters at the tip) and rotated toward the lesser curve by a radial motion of the handle. Slight opening and closing of the tissue mold (no more than 2-3 $\mathrm{mm}$ ) during this rotation allows the fundus to slide through the tissue mold and rotate around the esophagus. The external handle is rotated 120 to 180 degrees during this maneuver.

The Bell Roll maneuver results in pulling the lip of the GE valve caudally while rotating the fundus around the esophagus anteriorly and apposing the fundus to the esophagus in that rotated position. The tissue mold ends up at approximately the 1 o'clock position.

Failure to desufflate the stomach during this maneuver will greatly limit the amount of tissue that can be incorporated into the fundoplication. 

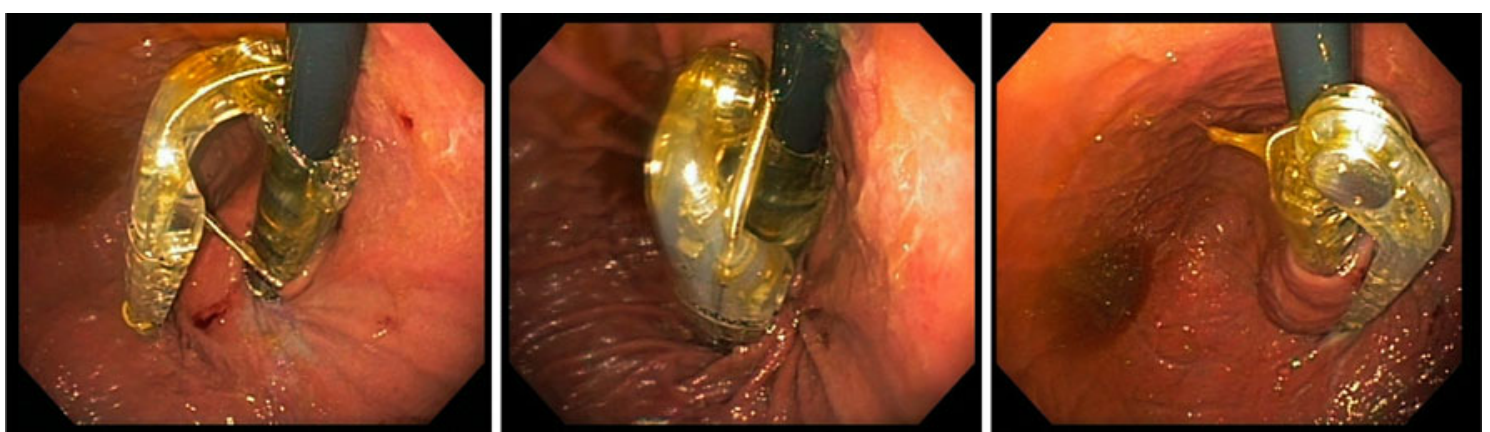

Fig. 9 Bell Roll maneuver. With the helix engaged at 12 o'clock and the tissue mold at 6 o'clock (left), the stomach is desufflated while the tissue mold is being rolled toward the 12 o'clock position (middle).

(The surgeon can compare this to attempting a laparoscopic fundoplication with a distended fundus.)

c. After completing the maneuver, the helical retractor and the tissue mold are locked in place before any radially directed tension on the handle is released. Additionally, suction is applied to the tissue invaginator and $30 \mathrm{~s}$ are allowed to elapse before tension is released on the device. The stomach is then reinsufflated. (The actions of locking the helical retractor and tissue mold, placing the tissue on suction, and gastric desufflation/insufflation may be performed best by the endoscopist, because the surgeon's hands are busy holding tension on the helical retractor, closing the tissue mold, and applying radial torque to the handle.)

d. If the external mark (bright tape on the shaft) corresponding to the previously determined location of the hiatus is outside of the mouth more than $1 \mathrm{~cm}$, the device is advanced (with suction still applied to the tissue invaginator) until the mark is within $1 \mathrm{~cm}$ of the mouth. This helps ensure that the esophagogastric plication is in an intra-abdominal position. (The diaphragmatic crura are approximately $1 \mathrm{~cm}$ cranial to the endoscopic location of the diaphragm with a fully reduced hernia based on intraoperative laparoscopy, which is why a $1-\mathrm{cm}$ gap is allowed.) If advancing the device this far leads to caudal slippage of the device, fastener deployment is only performed if other anatomic landmarks clearly indicate that the plication will be subdiaphragmatic.

e. The device will likely need to be rotated counterclockwise before stylet deployment for two reasons:

1. The torque applied to push the gastric fundus into the corner may lead to misalignment of the
This maneuver rolls fundus over and around distal esophagus to the 1 o'clock position (right)

tissue mold. This can be observed by rotating the endoscope to obtain a side view of the most posterior part of the mold; if it seems to be lifting out, it is misaligned. Counter-rotation realigns the mold and allows correct advancement of the stylet.

2. This counter-rotation brings the device out of the corner to allow more room to advance the stylet safely.

f. The posterior stylet (the one farthest from the corner) is advanced.

g. The H-fastener is deployed over the stylet. Experience indicates that when advancement of the $\mathrm{H}$ fastener meets resistance, it is helpful to pull back on the stylet a few millimeters and then advance both the fastener and stylet simultaneously.

h. The posterior stylet is withdrawn. With persistent counter-rotation and assurance that the device is advanced to the level of the hiatus, the anterior stylet is advanced and the anterior $\mathrm{H}$-fastener is deployed.

i. The device is reloaded with the tissue mold closed and the tissue invaginator on. This allows extra pressure for hemostasis and keeps the device in one location while attention is focused externally on reloading.

j. The helical screw is unlocked, the tissue invaginator is taken off suction, and the tissue mold is unlocked. This completes one rotational plication set, and if properly performed, two plications will be seen indenting the gastric mucosa.

$\mathrm{k}$. The device is rotated back toward 6 o'clock and two more rotational anterior plication sets at different plication depths are created using the above movements. This will result in six fasteners being deployed between 1.5 and $2.5 \mathrm{~cm}$ above the $\mathrm{Z}$-line, with the most anterior bringing the stomach 

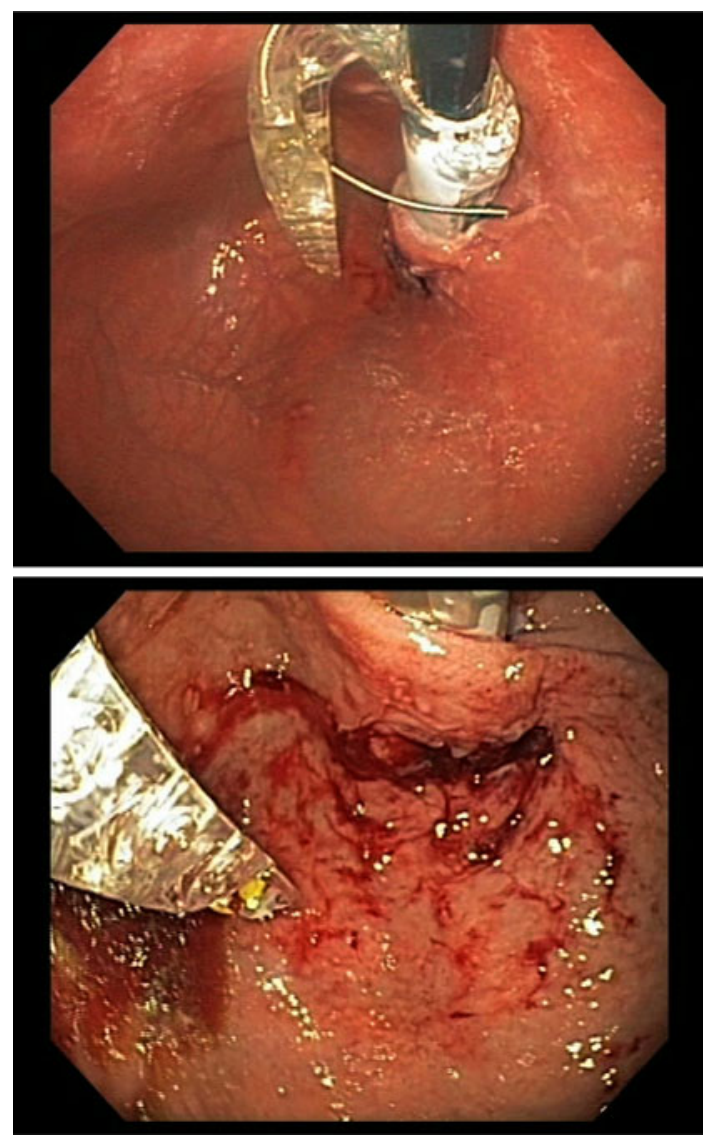

Fig. 10 Anterior rotational plication in two patients illustrating gradual advancement of gastric fundus toward lesser curve 1:30 position

up around the esophagus to the 1 o'clock to $1: 30$ position (Fig. 10).

II. Posterior rotational plication sets:

The device is now rotated through the lesser curve without releasing the helical screw from its 12 o'clock position, and three similar posterior rotational plication sets $1-2.5 \mathrm{~cm}$ above the Z-line are performed to create a posterior groove.

a. With the device unlocked and suction turned off, the device is introduced far enough so that the tissue mold in the nearly closed position has enough clearance to rotate counterclockwise through the lesser curve to the posterior position. The helical screw is left engaged at 12 o'clock, and the cable for the helical screw is advanced to eliminate tension on the helix as the device is rotated.

b. The tissue mold is placed at the 8 o'clock position and the set of simultaneous movements (described in step Ib) as the "Bell Roll maneuver," is performed, only with rotation clockwise (screen view).
1. The device is pulled back (cranially) a few centimeters and rotated clockwise toward the lesser curve as it is closed.

2. Tension is applied to the helical retractor.

3. The stomach is desufflated, allowing the tissue mold to reapproximate fundus to esophagus above the Z-line.

Performing these three movements results in pulling the lip of the GE valve caudally while folding the fundus against the esophagus and actually rotating the fundus around the esophagus posteriorly. The tissue mold is likely to end up at the 11 o'clock position.

c. Steps Ic to Ik described earlier for the anterior rotational plication sets are repeated in this posterior position, with the difference that the device is rotated counterclockwise. The anterior stylet is advanced first, and the second set of plications is slightly more posterior (toward lesser curve) than the first.

d. Three posterior rotational plication sets at different plication depths are created (Fig. 11). These often are at the same plicator position radially because the peritoneal reflection from the preaortic fascia to the pars flaccida of the stomach runs linearly. In this posterior position, the depth and ease of closure are limited by the diaphragmatic crura. In some patients, especially those with a hiatal hernia, the tissue mold may close inside the crura.

III. Anterior corner longitudinal plication sets

Frequently the creation of the above-described rotational plication sets does not fully plicate the anterior lesser curve. The esophagus courses anteriorly as it exits the diaphragm. Therefore, a gap may be seen on the anterior corner due to the device's straight shape and its ability to exert posterior pressure on the esophagus. This gap in the anterior lesser curve is more obvious after the posterior rotational plication sets are placed, and therefore it is addressed after the posterior plication sets.

a. The tissue mold is then rotated through the lesser curve again.

b. The anterior lesser curve of the valve (generally 12:30 to 2 o'clock positions) is built up by applying gentle caudal tension to the helical retractor and moving the tissue mold cranially during gastric desufflation. The ventral diaphragm runs at right angles to the esophagus and so limits the degree to which the tissue mold can be pulled cranially, and also limits the 

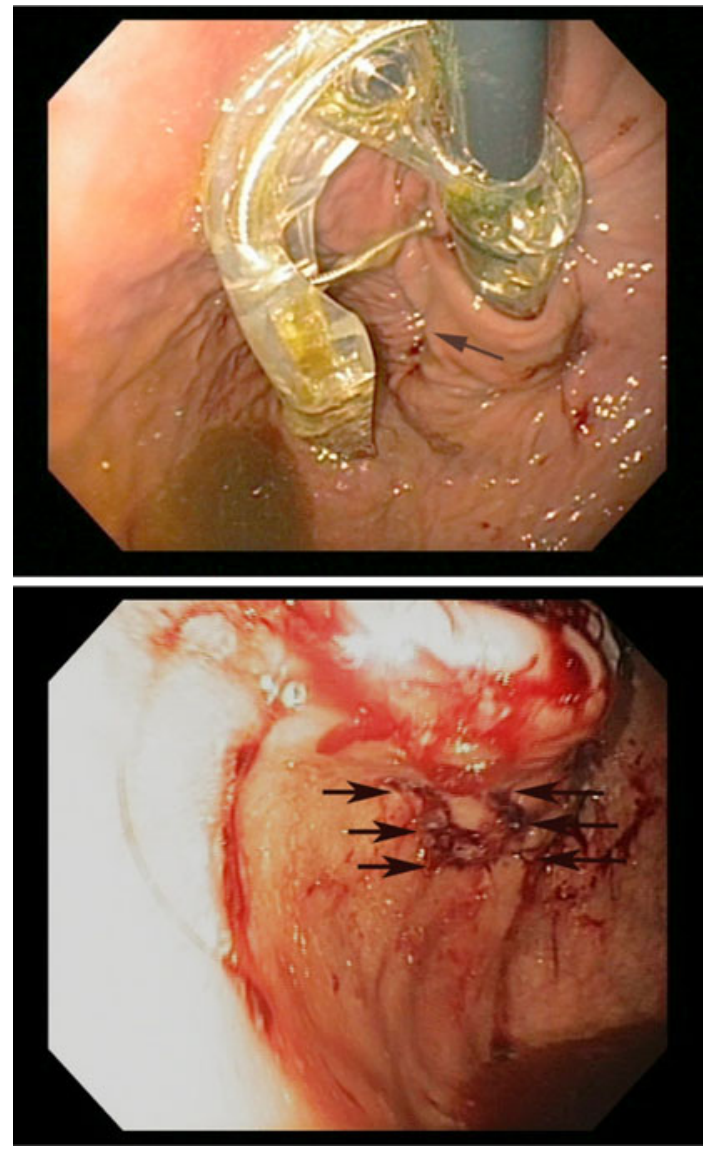

Fig. 11 Posterior rotational plication sets (arrows)

amount of tissue that can be gathered with the stomach insufflated. Gastric desufflation is therefore important if one wishes to perform these plications at an adequate depth. Occasionally, the tissue mold may pop inside the hiatus when the stomach is fully desufflated; this is generally not of concern if the gap at the hiatus is minimal $(<1 \mathrm{~cm})$.

c. Two plication sets at different plication positions (e.g., 12:30 and 1:30) at 1-2 cm plication depth are created (Fig. 12).

These are mostly longitudinal plications; there are times, however, when some element of rolling is possible. These sets especially should be performed gently as the esophageal wall is thinner here and there is already some degree of fixation due to the initial anterior rotational plications.

IV. Greater curve longitudinal deep plication set

The goal of this plication set is to fix the fundus to the esophagus at the greater curve position, thereby providing length to the valve.

a. The helical screw at the 12 o'clock position provides little benefit for this plication set

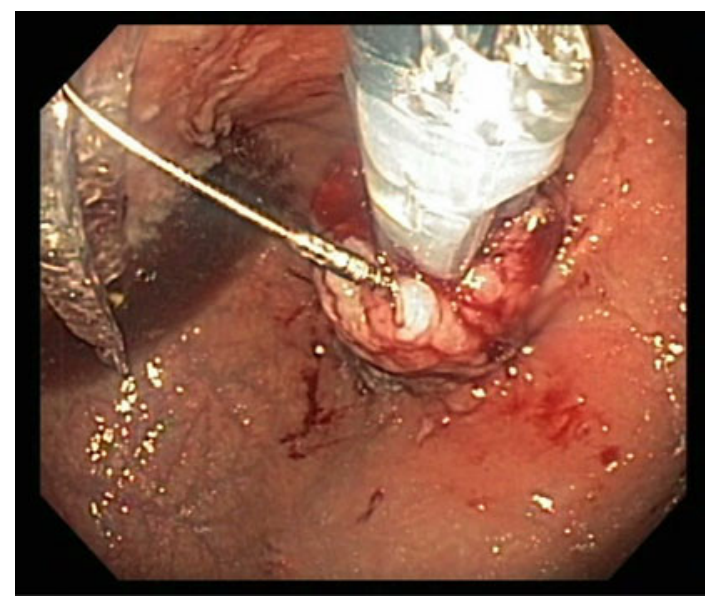

Fig. 12 Anterior longitudinal plication sets from the 12:30 to 2 o'clock positions

because it is diametrically opposite. Therefore, it is released and then redeployed at the Z-line at the greater curve (between 5 o'clock and 6 o'clock; Fig. 13).

b. One to two plication sets are performed at roughly 5 o'clock at a plication depth of up to $3.5 \mathrm{~cm}$, depending on anatomic issues described below.

c. At the greater curve position, the diaphragm may get incidentally incorporated into the tissue mold, which is not desirable (Fig. 13). To avoid that, the following precautions should be observed:

1. Be sure the device is introduced to the level of the hiatus before stylet deployment.

2. Use the stylet to gauge the thickness of the incorporated tissue. The tip of the stylet should

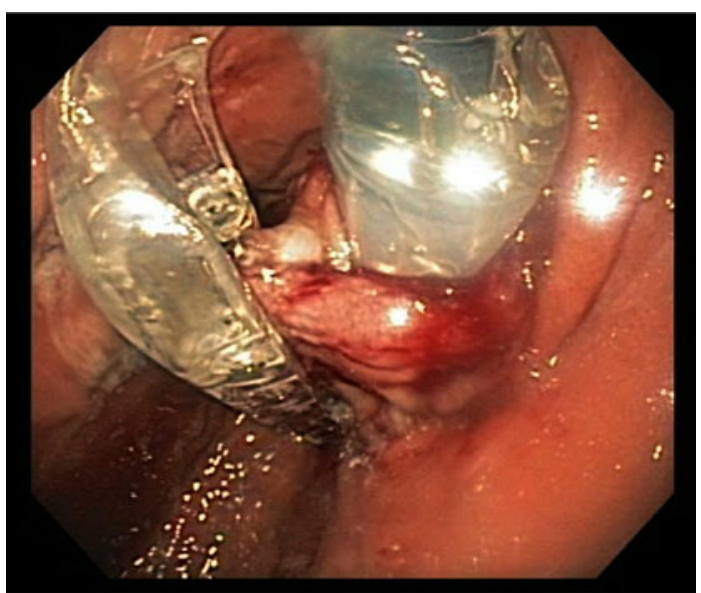

Fig. 13 With caudal tension on the helical retractor, which is now at 6 o'clock, the tissue mold is closed to create a longitudinal deep plication set at 6 o'clock. This is the area where it is easiest to inadvertently incorporate diaphragm in the plication 
be visible beyond the tissue mold before the lever on the handle reaches midway along its travel path. This indicates that an appropriate and not excessive amount of tissue is incorporated with the fasteners. If too much tissue is incorporated, it may indicate that this includes the diaphragm, and excess tension may result with the potential for disruption of the esophageal or gastric wall.

3. Excessive pressure during fastener deployment may indicate that the plicator is being pushed through diaphragm or crura, because these are tougher than the esophageal or gastric wall.

4. Opening and closing the tissue mold as it is pulled cranially enables the operator to palpate the diaphragm through the gastric fundus and is better to determine the anatomic relationships. Keeping the tissue mold almost closed while positioning the device before fastener deployment also will lessen the potential for incorporating the diaphragm.

V. Final view

The resulting fundoplication has a classic omega appearance and can be compared with the initial preoperative retroflexed view (Fig. 14).

\section{Number of fasteners to deploy}

The device uses cartridges with 20 fasteners each. The goal of our technique is to deploy all 20 fasteners as 10 plication sets: 3 anterior rotational sets toward 1 o'clock; 3 posterior rotational sets toward 11 o'clock; 2 anterior longitudinal sets between 12 o'clock and 2 o'clock; and 1 longitudinal at 6 o'clock; the remaining 1 plication is set at 6 o'clock or as a "cleanup" fixation where the fastener may not have deployed well the first time (Fig. 15). With appropriate technique, $90 \%$ of fasteners can be properly deployed. We use fasteners until we have at least 18 satisfactory deployments.

\section{Technique of fastener deployment}

As the fastener is advanced, the operator encounters the first resistance when the leading leg of the fastener comes out of the device into the esophageal tissue. Partial withdrawal of the stylet and then advancement of both the stylet and the fastener together ("shuffle technique") greatly decreases the resistance and aids in smooth deployment. After a few millimeters of advancement, the trailing leg begins to engage within the esophageal lumen. A gentle pause or slow advancement leads to a more satisfactory trailing leg deployment. The fastener is then advanced over
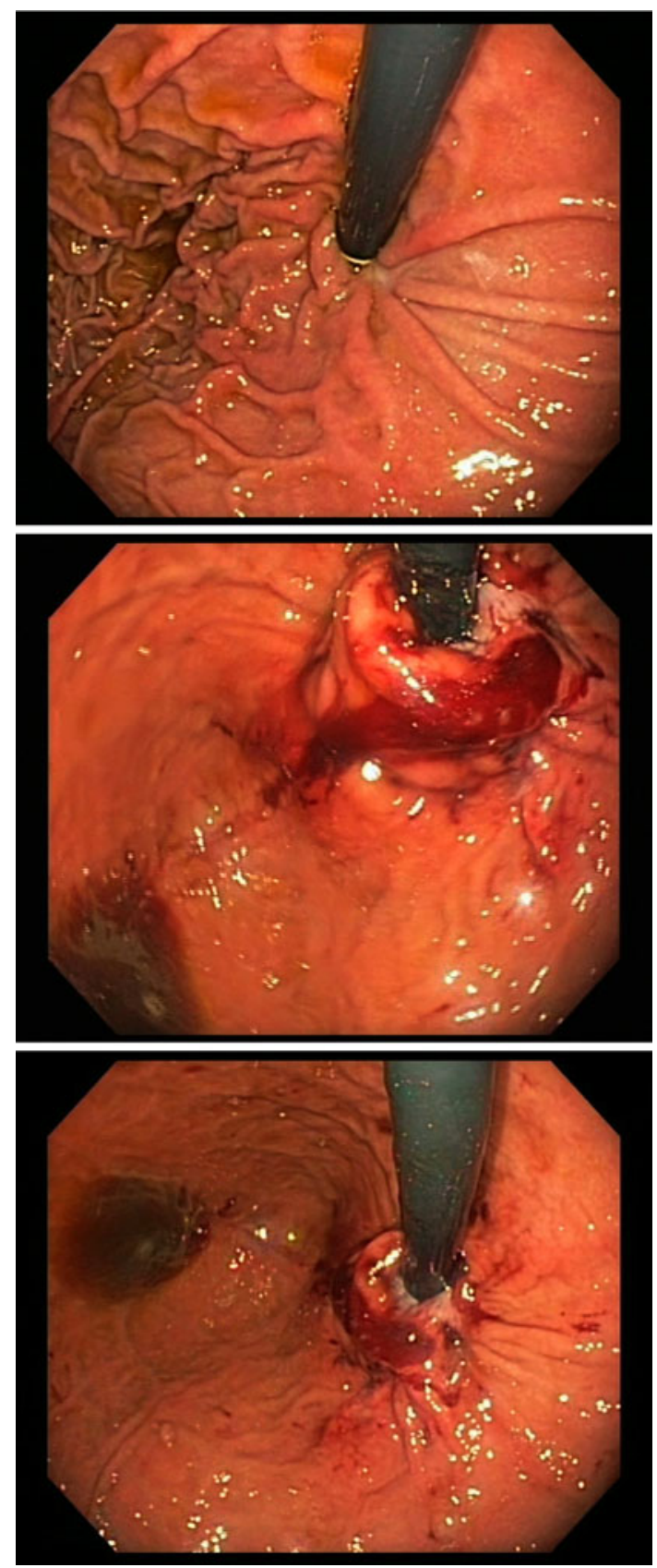

Fig. 14 Preoperative and postoperative appearance of the gastroesophageal junction on retroflex endoscopy

the stylet. If the resistance is noted again, the shuffle technique is employed again. Once the tip of the fastener becomes visible at the tissue mold, the stylet is pulled back so that the arrowhead of the stylet helps derail the leading leg as it is advanced slowly.

\section{Fastener misfires}

Fasteners may misfire by being deployed prematurely, or when the trailing leg fails to engage. When the trailing leg 


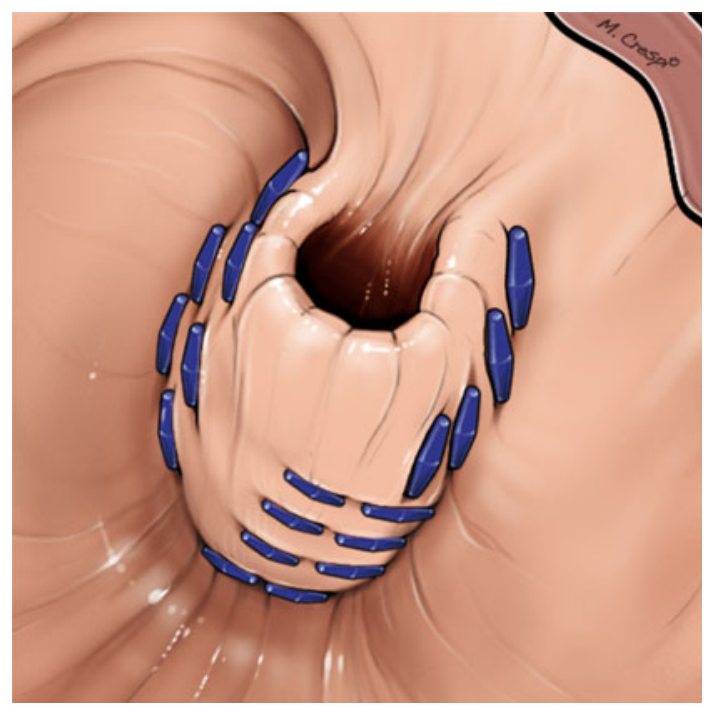

Fig. 15 Illustration of postoperative view with positions of various plication sets

fails to engage, the whole fastener follows the stylet path and falls into the gastric lumen. In this situation, repeated loading and firing of the stylet may lead to an esophageal laceration because the trailing leg is less likely to engage an enlarging hole. We therefore always reposition the device completely in cases where the fastener does not deploy properly.

\section{Withdrawal and final endoscopy}

Once the esophagogastric plication has been created to the surgeon's satisfaction, the device is straightened (un-retroflexed) under endoscopic visualization and, ensuring that the helical screw is returned to its groove in the tissue mold and pulled cranially, the device is withdrawn with the endoscope just inside the device so that fasteners can be observed during withdrawal. Immediate repeat endoscopy is performed to evaluate the esophageal lumen to ensure that there is no laceration or bleeding and to evaluate the plication in retroflex view for bleeding. Endoclips should be available to treat laceration or bleeding.

\section{Postoperative care}

Patients experience epigastric, chest, and shoulder pain and may require narcotic pain medication for 3-4 days after surgery. Recognizing the limitations of radiographic swallows at detecting esophageal leaks, routine use of contrast studies, and checking white blood cell count may be of benefit, and we employ them routinely. Pain beyond 4 days is unusual and should prompt consideration of esophageal or gastric leak. CBC, CT scan, and water-soluble esophageal swallow should be performed as clinically warranted.

\section{Decreasing potential for and management of complications}

Following are considerations for decreasing potential complications:

a. Use the hiatal landmark. At intraoperative endoscopy before the device introduction, measure the distance from incisors to the hiatal landmark. Then, during the procedure, after locking the helix and tissue mold and placing the tissue invaginator on suction for $30 \mathrm{~s}$, the EsophyX device is advanced to this measured distance before advancing the stylet. (The device has external numbering corresponding to centimeters from the stylet orifice.)

b. Use the stylet as a rough gauge of tissue thickness. In general, we have found that the stylet should be visible at the edge of the tissue mold by the time the lever is halfway along its path on the handle. This corresponds to approximately $6 \mathrm{~mm}$ of tissue within the tissue mold.

c. Be continuously aware of the anatomy external to the stomach, especially the diaphragm in relation to the fundus.

d. Only deploy the stylet and fastener once in any given location. If the fastener does not deploy properly, do not reload the fastener and try to deploy a second fastener in the same location, because this may widen the tract and increase the potential for a leak.

e. Consider prophylactic antibiotics for the procedure as transmural fixation is employed.

f. Consider continuing PPI therapy for 10-14 days after the procedure to decrease the risk of bleeding from the stomach side of the fundoplication.

g. Reduce the number of helical retractor deployments to one at the 12 o'clock position and, if needed, a second one at the greater curve. This not only increases the efficiency of the procedure but also limits trauma to the GE valve.

Following are consideration for the management of complications:

a. As with any minimally invasive GI procedure, complications can still be major. A low threshold for investigation and reoperation are appropriate.

b. Endoclips may be successfully deployed through the 2.8-mm working channel of the flexible endoscope to reapproximate esophageal mucosa over a laceration, or even a very deeply seated fastener.

c. Bleeding from the wrap is generally at the site of the helical fastener. Endoclips may be successfully deployed in retroflex position to manage bleeding from the edge of the plication. 
d. Pain that persists beyond 4 days or is increasing in severity, fever, or malaise within the first 10 days of surgery should be promptly investigated. CT scan of the chest and abdomen and/or contrast GI series should be considered.

e. If mediastinal abscess is diagnosed, laparoscopic dissection of the hiatal attachments, localization of the perforation by air insufflation in the esophagus, removal of problematic fasteners, closure of the perforation, and a Thal patch can be combined with transhiatal debridement and transabdominal external drainage of the abscess. Creation of a traditional partial fundoplication rather than circumferential fundoplication may be preferable if it is deemed appropriate to create a sutured fundoplication after treatment of the perforation [6].

\section{Clinical experience}

Between November 2008 and September 2010, we performed over 100 TIF fundoplications during which this technique evolved. Mean operative time was 75 (range 50-94) min in our first 30 patients, and now averages $55 \mathrm{~min}$. Postoperative stay was typically 1 day as was the duration of IV pain medication. Immediate postoperative complications ( $<7$ days after the procedure) included one patient with a GI bleed requiring transfusion, and two patients with a mediastinal abscess that required laparoscopic drainage and closure. Traumatic dislodgement of the helical screw was the etiology of the bleeding in the third patient in our series; since that time we routinely unlock the helical screw as the first maneuver after fastener deployment, and tighten the helical screw between each positioning. We have not experienced bleeding issues since then. Both patients with a mediastinal abscess developed a leak from anterior fasteners that traversed the diaphragm in their course from esophagus to fundus. One patient presented 6 days after the procedure and required laparoscopy with drainage and creation of a posterior Toupet fundoplication (no visible leak at operation). The other patient required laparoscopic drainage, suture closure or the perforation, and a Thal patch $24 \mathrm{~h}$ after the initial TIF. Both patients recovered uneventfully. These two leaks led us to thoroughly review our technique in relation to anatomy, and the techniques described herein are the result of that review.

Disclosures Dr. Bell and Dr. Cadiere are on the speaker's bureau for EndoGastric Solutions.

Open Access This article is distributed under the terms of the Creative Commons Attribution Noncommercial License which permits any noncommercial use, distribution, and reproduction in any medium, provided the original author(s) and source are credited.

\section{References}

1. Cadiere GB, Buset M, Muls V, Rajan A, Rosch T, Eckardt AJ, Weerts J, Bastens B, Costamagna G, Marchese M, Louis H, Mana F, Sermon F, Gawlicka AK, Daniel MA, Deviere J (2008) Antireflux transoral incisionless fundoplication using EsophyX: 12-month results of a prospective multicenter study. World J Surg 32:1676-1688

2. Cadiere GB, Rajan A, Germay O, Himpens J (2008) Endoluminal fundoplication by a transoral device for the treatment of GERD: a feasibility study. Surg Endosc 22:333-342

3. Cadiere GB, Van Sante N, Graves JE, Gawlicka AK, Rajan A (2009) Two-year results of a feasibility study on antireflux transoral incisionless fundoplication (TIF) using EsophyX. Surg Endosc 23:957-964

4. Bell RCW, Freeman KD (2010) Clinical and pH-metric outcomes of transoral esophagogastric fundoplication for the treatment of gastroesophageal reflux disease. Surg Endosc. doi:10.1007/ s00464-010-1497-9

5. Jobe BA, O'Rourke RW, McMahon BP, Gravesen F, Lorenzo C, Hunter JG, Bronner M, Kraemer SJ (2008) Transoral endoscopic fundoplication in the treatment of gastroesophageal reflux disease: the anatomic and physiologic basis for reconstruction of the esophagogastric junction using a novel device. Ann Surg 248: 69-76

6. Killic A, Schuchert MJ, Luketich J, Landreneau RJ (2009) Surgical management of epiphrenic diverticula in the minimally invasive era. JSLS 13:160-164 